OPEN

SUBJECT AREAS:

ANTIVIRALS

VIRAL INFECTION

Received

28 March 2014

Accepted

6 June 2014

Published

26 June 2014

Correspondence and requests for materials should be addressed to K.Z. (keivan@um.edu.

my)

\section{Baicalin, a metabolite of baicalein with antiviral activity against dengue virus}

\author{
Ehsan Moghaddam', Boon-Teong Teoh', Sing-Sin Sam', Rafidah Lani', Pouya Hassandarvish', \\ Zamri Chik², Andrew Yueh'3 ${ }^{3}$ Sazaly Abubakar' \& Keivan Zandi'
}

\begin{abstract}
${ }^{1}$ Tropical Infectious Disease Research and Education Center, Department of Medical Microbiology Faculty of Medicine, University of Malaya, 50603 Kuala Lumpur, Malaysia, ${ }^{2}$ Department of Pharmacology, Faculty of Medicine, University of Malaya, 50603 Kuala Lumpur, Malaysia, ${ }^{3}$ Institute of Biotechnology and Pharmaceutical Research, National Health Research Institutes, Miaoli 350, Taiwan, ROC.
\end{abstract}

Baicalin, a flavonoid derived from Scutellaria baicalensis, is the main metabolite of baicalein released following administration in different animal models and human. We previously reported the antiviral activity of baicalein against dengue virus (DENV). Here, we examined the anti-DENV properties of baicalin in vitro, and described the inhibitory potentials of baicalin at different steps of DENV-2 (NGC strain) replication. Our in vitro antiviral experiments showed that baicalin inhibited virus replication at $\mathrm{IC}_{50}=13.5$ $\pm 0.08 \mu \mathrm{g} / \mathrm{ml}$ with $\mathrm{SI}=21.5$ following virus internalization by Vero cells. Baicalin exhibited virucidal activity against DENV-2 extracellular particles at $\mathrm{IC}_{50}=8.74 \pm 0.08 \mu \mathrm{g} / \mathrm{ml}$ and showed anti-adsorption effect with $\mathrm{IC}_{50}=18.07 \pm 0.2 \mu \mathrm{g} / \mathrm{ml}$. Our findings showed that baicalin as the main metabolite of baicalein exerting in vitro anti-DENV activity. Further investigations on baicalein and baicalin to deduce its antiviral therapeutic effects are warranted.

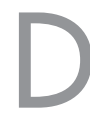

engue virus (DENV) is an enveloped RNA virus belonging to the Flaviviridae family. There are four distinct serotypes, DENV-1, DENV-2, DENV-3, and DENV-4. Dengue virus can cause a range of diseases from asymptomatic infection to mild dengue fever (DF) or severe dengue hemorrhagic fever (DHF) and dengue shock syndrome (DSS) ${ }^{1,2}$. DENV is transmitted principally in a cycle that involves humans and mosquito vectors, Aedes aegypti and Aedes albopictus. All DENV serotypes are widespread geographically in tropical and subtropical regions of the world, causing life-threatening disease imposing considerable health and economic burden. Currently, there is no approved vaccine or antiviral agents against clinical dengue necessitating prompt strategies to design effective antiviral strategies against this infection.

In recent years, many investigators focus on plants and their derivatives to develop new antiviral drugs ${ }^{3-5}$. Some phytochemicals have been shown to have therapeutic applications against genetically and functionally diverse viruses $^{6,7}$. Flavonoids are polyphenolic plant metabolites with numerous biological activities and low toxicity. More than 5000 natural flavonoids have been identified in plants or various dietary sources that are presumed to have potential health benefits ${ }^{8}$. Antiviral properties of flavonoids, a group of plant polyphenolics have been reported against different viruses including $\mathrm{DENV}^{3,9-11}$. Recently, we showed that baicalein, a flavonoid belonging to the flavones subgroup (Figure 1A) exhibited significant antiviral effects against in vitro replication of DENV-2 in Vero cells, functioning at different stages of virus replication' ${ }^{9}$. Baicalein (5, 6, 7-trihydroxyflavone) is a flavonoid originally isolated from Scutellaria baicalensis, a Chinese medicinal plant with various biological properties. Baicalin (5,6-dihydroxy-7-O-glucuronide flavone) is also a flavonoid (Figure 1B) presents in the roots of S.baicalensis Baicalein is metabolized and converted mainly to baicalin following intake to animals and humans ${ }^{12-16}$.

It has also been shown that $\sim 90 \%$ of baicalein administered is metabolized to baicalin ${ }^{17}$, and hence, it is necessary to investigate the role of baicalin in any particular medical condition.

Here, we determined the antiviral activities of baicalin at different stages of DENV replication in Vero cells and on DENV replicon cell line. We showed that baicalin interferes and inhibits DENV-2 in vitro replication at various stages of the virus replication cycle.

\section{Results}

Cytotoxic activity of baicalin. Cytotoxicity assay was performed to determine the non-toxic concentrations of baicalin against Vero cells using the MTS assay. We found that the half maximal cytotoxic concentration $\left(\mathrm{CC}_{50}\right)$ 
<smiles>O=c1cc(-c2ccccc2)oc2cc(O)c(O)c(O)c12</smiles>

(A)<smiles>O=C(O)C1O[C@@H](Oc2cc3oc(-c4ccccc4)cc(=O)c3c(O)c2O)[C@H](O)[C@@H](O)[C@@H]1O</smiles>

(B)
Figure 1 Chemical structure of baicalein (A) and baicalin (B).

of baicalin was $290.9 \mu \mathrm{g} / \mathrm{ml}$. At a concentration of $62.5 \mu \mathrm{g} / \mathrm{ml}$, the viability of baicalin-treated Vero cells was $>90 \%$ compared to vehicle control indicating that the concentration could be considered as the maximum non-toxic dose (MNTD) of baicalin for Vero cells (Figure 2). Therefore, $50 \mu \mathrm{g} / \mathrm{ml}$ was chosen as the highest concentration of baicalin in all antiviral assays that was lower than the maximum non-toxic dose MNTD of baicalin.

Inhibitory effect of baicalin on BHK-DENV replicon cell line. To determine whether baicalin exerts antiviral functions at the intracellular stages of DENV-2 replication, we studied the effect of the compound on BHK-DENV subgenomic replicon cell line that encodes only non-structural viral proteins. Our results showed that baicalin inhibited replication of DENV subgenomic replicon (Figure 3). Baicalin exhibited a significant antiviral activity with $\mathrm{IC}_{50}=14.9 \mu \mathrm{g} / \mathrm{ml} \pm 0.07$ against intracellular DENV-2 intracellular replicon by targeting non-structural protein(s) of virus. This observation corroborated the data obtained in the time-of-drugaddition studies.

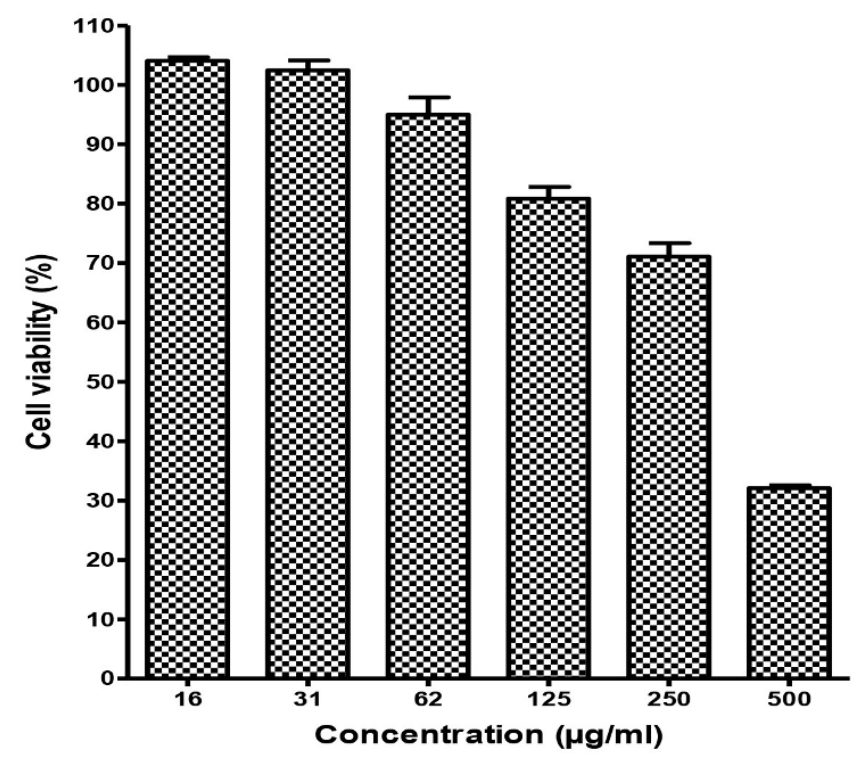

Figure 2 Cytotoxicity of baicalin against Vero cells. MTT assay was used to evaluate the cytotoxicity of the baicalin. Different concentrations of baicalin up to $500 \mu \mathrm{g} / \mathrm{ml}$ were used to treat the Vero cells for 4 days. Data showed that the Vero cells viability after 4 days using The MNTD of baicalin is $62 \mu \mathrm{g} / \mathrm{ml}$ is more than $90 \%$.Therefore, the MNTD value of baicalin is $62 \mu \mathrm{g} / \mathrm{ml}$. All experiments were conducted as three independent experiments in triplicates and the data were plotted using Graph Pad Prism Version 5 (Graph Pad Software Inc., San Diego, CA.).

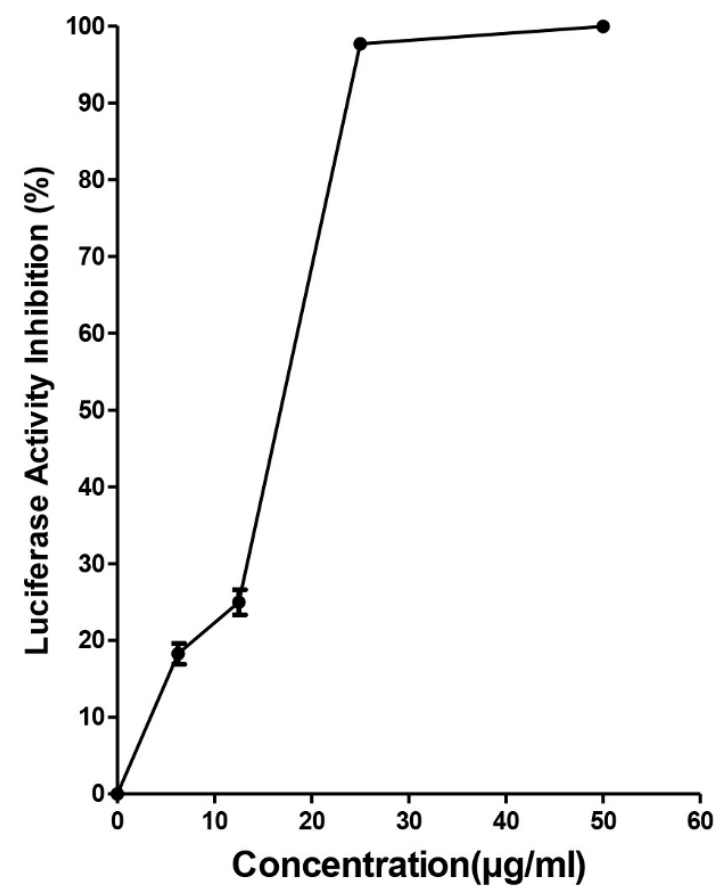

Figure 3 Evaluation of anti-dengue activity of baicalin using BHKDENV replicon cell line. Luciferase activity of BHK-DENV replicon cells after $48 \mathrm{~h}$ of culture in the presence of increasing concentrations of baicalin up to the $50 \mu \mathrm{g} / \mathrm{ml}$ were measured. All data were normalized to the DMSO control. Data from triplicate assays for three independent experiments were plotted using Graph Pad Prism Version 5 (Graph Pad Software Inc., San Diego, CA.).

Baicalin inhibits DENV-2 replication in vitro in a dose-dependent manner. The in vitro anti-DENV activity of baicalin was evaluated by DENV foci reduction assay and virus yield reduction assay. We observed that baicalin inhibited DENV-2 infectious foci in a dosedependent manner (Figure 4A). At $50 \mu \mathrm{g} / \mathrm{ml}$ of baicalin, DENV-2induced foci formation was completely inhibited (Figure 4C). The antiviral effect of baicalin was further confirmed by virus yield reduction assay using q RT-PCR (Figure 4B). On the first day post infection the DENV RNA copy number was dropped from 609 copies (non-treated DENV infected cells) to 106 copies in the treated cells with $50 \mu \mathrm{g} / \mathrm{ml}$ of baicalin which is equal to $72.2 \% \pm$ 0.5 inhibition of virus yield production (Figure $4 \mathrm{~A}$ ). Interestingly, in the second day post infection the copy number of DENV RNA in non-treated wells (15638 copies) has dropped to 1829 copies in the presence of $50 \mu \mathrm{g} / \mathrm{ml}$ of baicalin $(78.3 \% \pm 0.7$ inhibition). The result of virus yield assay for the third day post infection showed the significant DENV replication inhibition by dropping the DENV RNA copy number from 422905 copies in the non-treated cells to 10572 copies in the treated cells with $50 \mu \mathrm{g} / \mathrm{ml}$ of baicalin, equal to $97.52 \% \pm 0.5 \mathrm{DENV}$ replication inhibition (Figure 4C).

Baicalin exerts virucidal activity against free DENV-2 particles. We have previously shown that baicalein exerts significant virucidal activity against extracellular DENV-2 particles ${ }^{3}$. Hence, in the present study we examined whether baicalin, the main metabolite of baicalein could also inactivate extracellular DENV-2 particles and prevent subsequent infection. Baicalin was pre-incubated with the virus suspension and diluted to sub-therapeutic concentrations prior to infecting the respective host cell. Our results showed that baicalin inactivated DENV-2 virions (Figure 5), and importantly, baicalin exhibited a maximum function at an $\mathrm{IC}_{50}$ of $8.74 \pm 0.08 \mu \mathrm{g} / \mathrm{ml}$. However, it was shown that $25 \mu \mathrm{g} / \mathrm{ml}$ of baicalin which was lower than MNTD of the compound was able to exhibit $96.1 \% \pm 1$ 


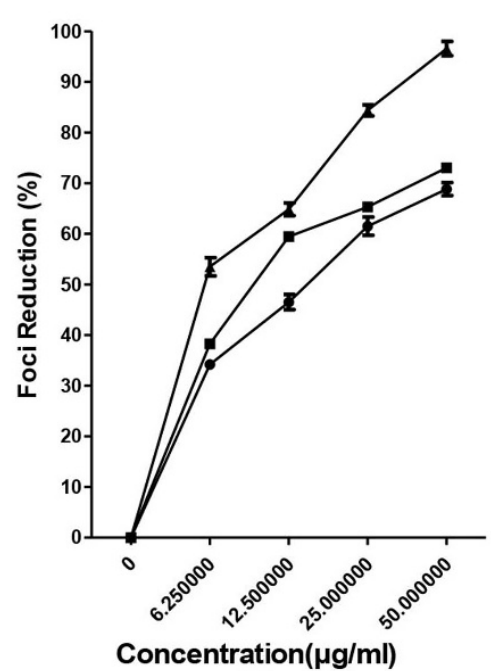

(A)

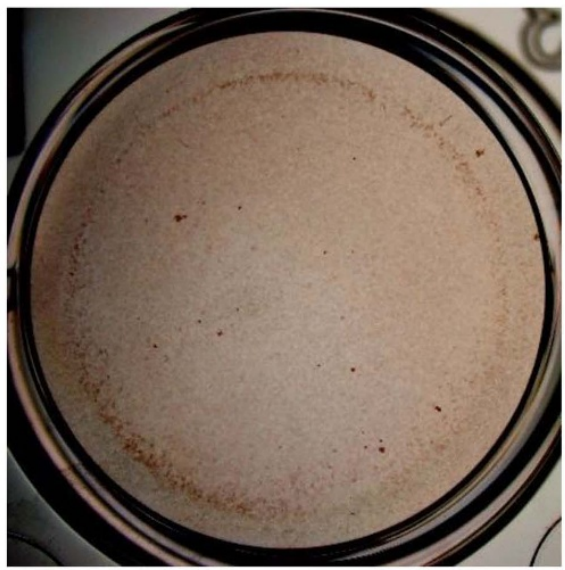

(C)
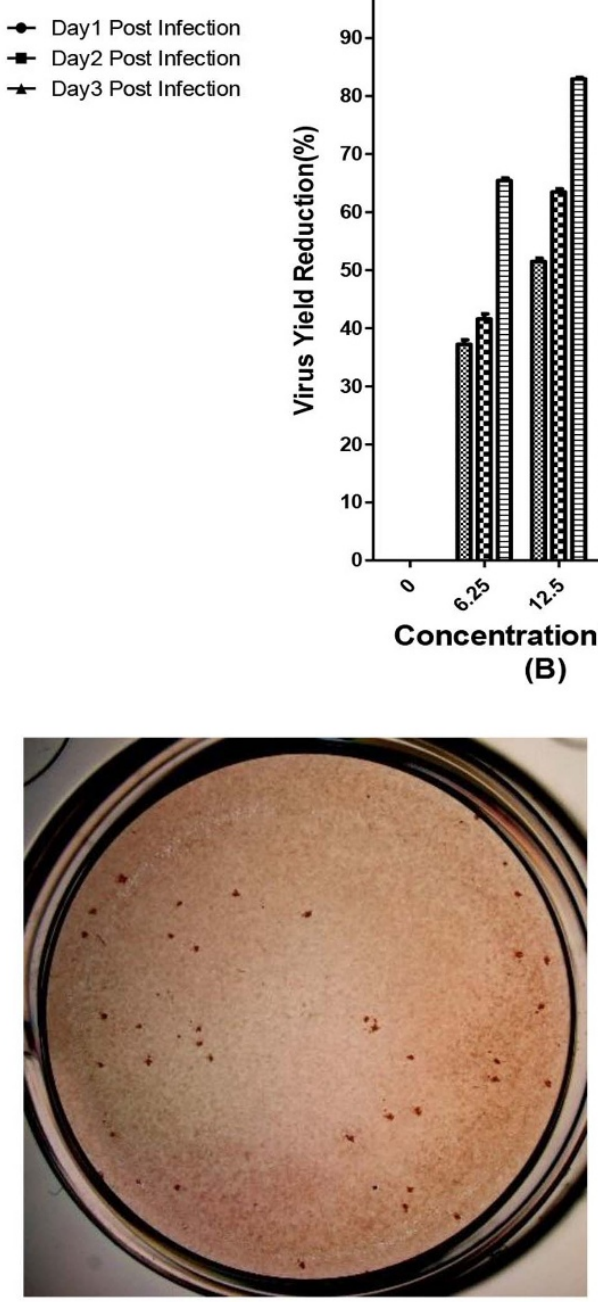

(D)

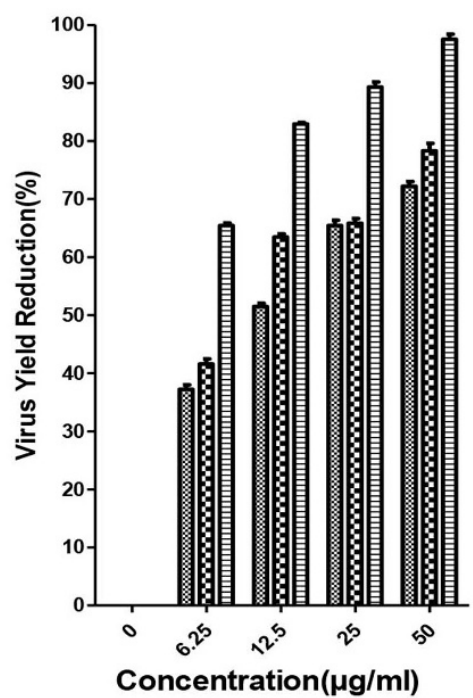

(B)
Day1 Post Infection $\infty$ Day2 Post Infection Day3 Post Infection

Figure $4 \mid$ Anti-dengue effect of continuous treatment with baicalin in Vero cells. Foci forming unit reduction assay (FFURA) was used to evaluate the in vitro antiviral activity daily up to three days post infection (A). Anti-dengue activity of $50 \mu \mathrm{g} / \mathrm{ml}$ of baicalin (C); or with $25 \mu \mathrm{g} / \mathrm{ml}$ of baicalin (D) or without baicalin (E) using DENV foci immunostaining method on the day three post infection were shown. The respective DENV-2 RNA copy number was quantified using qRT-PCR (B). DENV-2 in vitro replication is inhibited more than $90 \%$ using $50 \mu \mathrm{g} / \mathrm{ml}$ of baicalin which is lower than MNTD. The percentages of foci reduction (\% RF) and RNA copy number reduction were obtained by comparing against untreated controls maintained in parallel. Data from triplicate assays for three independent experiments were plotted using Graph Pad Prism Version 5 (Graph Pad Software Inc., San Diego, CA.).

reduction for DENV-2 infectious foci number (Figure 5). The data suggested that baicalin directly inactivates free DENV-2 particles and neutralize their infectivity attributes.

Baicalin exerts inhibitory effect by acting against DENV-2 cell attachment but not entry. Next, to further characterize the antiviral mechanism(s) of baicalin, we investigated the effect of the compound against virus attachment to host cell receptors. The change in temperature between $4^{\circ} \mathrm{C}$ (permitting virus binding but not entry) and $37^{\circ} \mathrm{C}$ (facilitating virus entry/penetration) allows examination of the effect of the drug on each specific cellular event. Our study showed that baicalin prevented attachment of DENV-2 to the Vero cells (Figure 6) at $\mathrm{IC}_{50}=18.07 \pm 0.2 \mu \mathrm{g} / \mathrm{ml}$. Figure $6 \mathrm{C}$ showed the $75.2 \% \pm 1.1$ reduction in number of DENV foci due to interruption with DENV attachment to the cells.

To further investigate whether baicalin retained its effects against cellular penetration stage of DENV-2, we facilitated viral binding to cell receptors at $4{ }^{\circ} \mathrm{C}$ followed by inactivation of unabsorbed viruses. Subsequently, the adsorbed viruses were facilitated to penetrate the host cell membrane by rendering a temperature shift to $37^{\circ} \mathrm{C}$ in the presence or absence of the baicalin. Our experiments did not reveal any significant activity by baicalin against penetration of attached DENV-2 to Vero cells (data not shown). Moreover, there was no significant inhibitory activity for baicalin against DENV-2 replication efficiency when it was used in pre-treatment mode (data not shown).

Antiviral activity of baicalin after virus entry to the host cell. To investigate the inhibitory effect of baicalin against intracellular DENV-2 replication, the infected Vero cells were treated with increasing concentrations of baicalin. Our investigation showed that baicalin at a concentration of $50 \mu \mathrm{g} / \mathrm{ml}$ inhibited DENV-2 replication to $>99 \%$ (Figure 7 ) with $\mathrm{IC}_{50}=13.50 \pm 0.08 \mu \mathrm{g} / \mathrm{ml}$.

\section{Discussion}

Dengue virus is the most common human arbovirus with $\sim 390$ million estimated cases per year and $\sim 96$ million symptomatic patients ${ }^{18}$. The greatest number of dengue cases occurs in Asia and Latin America, but the disease reportedly spreads across the different unaffected parts of the world due to various factors such as climate 


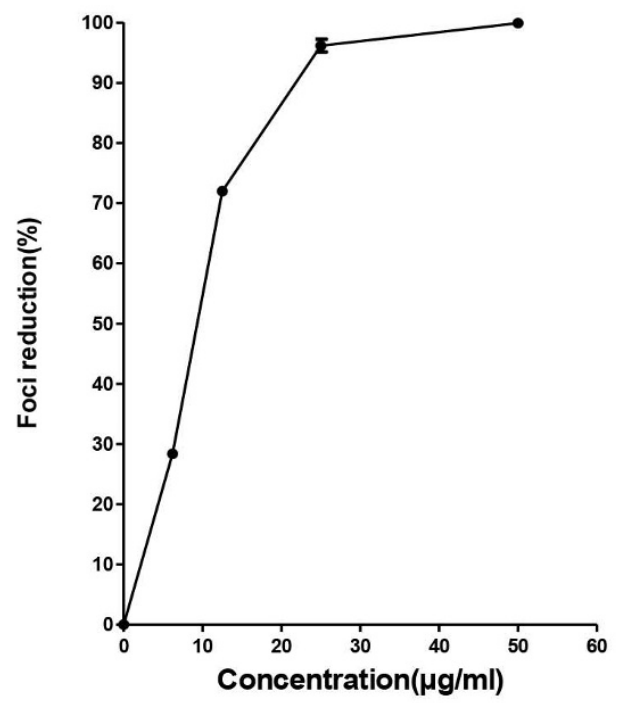

(A)

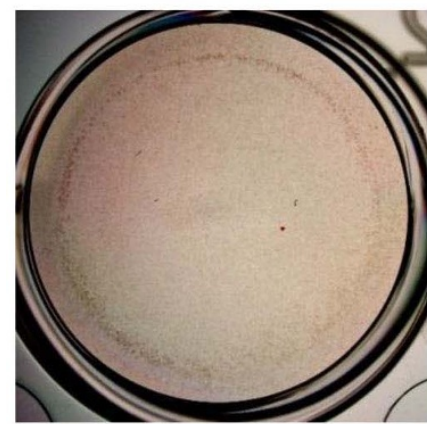

(B)

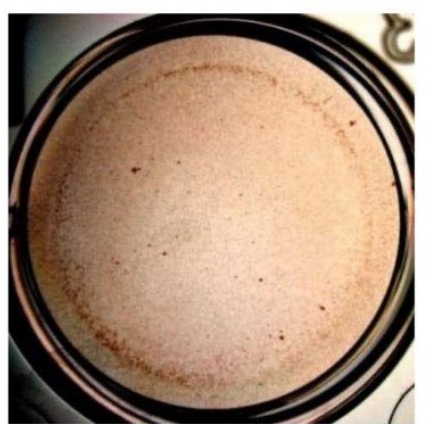

(C)

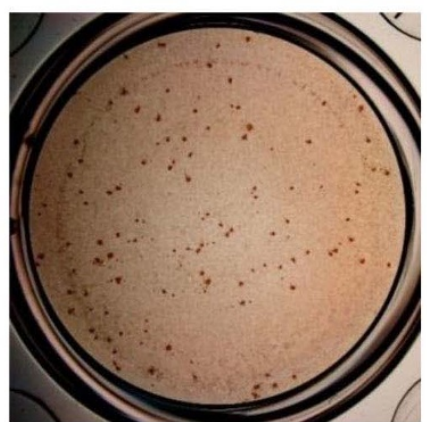

(D)

Figure 5 | Virucidal activity of baicalin on DENV-2 free particles. Foci forming unit reduction assay (FFURA) on Vero cells was performed to evaluate the virucidal activity of baicalin on DENV-2 extracellular particles. Virucidal activity of $50 \mu \mathrm{g} / \mathrm{ml}$ of baicalin (B); or with $25 \mu \mathrm{g} / \mathrm{ml}$ of baicalin (C) or without baicalin (D) using DENV foci immunostaining method on the day 4 post infection were shown. It is shown that $50 \mu \mathrm{g} / \mathrm{ml}$ of baicalin can inactivate more than $99 \%$ of DENV-2 free particles (A). Data from triplicate assays for three independent experiments were plotted using Graph Pad Prism Version 5 (Graph Pad Software Inc., San Diego, CA.).

change, increased travelling and unplanned urbanization ${ }^{19}$. To date, there is no licensed vaccine or effective antiviral therapy for prevention and treatment of dengue infection. Therefore, drug discovery against dengue virus remains a priority.

An efficient and safe antiviral agent for dengue virus should ideally have the potential to decrease the number of infected individuals with clinical presentations, and also protect travellers who travel to dengue endemic regions. Therefore, development of effective antidengue drug with the above mentioned criteria assumes significant priority $^{20}$. Among the different compounds with various biological benefits including antiviral activity, flavonoids represent the target of interest to drug discovery scientists ${ }^{21-23}$.

Baicalin, a flavonoid isolated mainly from the roots of S.baicalensis, is also the main metabolite of baicalein. In our previous study, we found that baicalein exerts antiviral activity against DENV-2 in Vero cells by different mechanisms ${ }^{3}$. We have also reported the anti-dengue activity of an extract of the roots of Scutellaria baicalensis as the main natural source for baicalein and baicalin against in vitro replication of dengue virus previously ${ }^{24}$. It was demonstrated that baicalein after administration to the different animals and human converts it to baicalin as its main metabolite $^{12,13,25}$. However, the conversion of baicalin to baicalein occurs during digestion by the removal of a glycoside moiety by $\beta$ - glucoronidase ${ }^{15,26}$. $\beta$-glucoronidase can be found as a lysosomal enzyme mainly available in the lumen of intestine and also produced by certain intestinal commensal bacteria ${ }^{15,27}$ but still there is no evidence related to that conversion in cell culture. Here, we report that baicalin, the main metabolite of baicalein inhibits the replication of DENV-2 in Vero cells through various mechanisms. In DENV-2 subgenomic replicon system, baicalin also showed inhibitory activity that indicates its credentials against DENV intracellular replication by potentially affecting viral genes. Our data from the replicon assay was in concordance with results of foci forming assay and virus-yield study. DENV yield in Vero cells was significantly reduced after exposing doses of baicalin lower than its MNTD.

Our studies showed that baicalin did not affect DENV replication when it was added to the Vero cells prior to virus infection. Thus, it is unlikely that it exerts antiviral activity by directly affecting the host's cell. We showed that baicalin affects DENV-2 replication when it was added after virus entry which is consistent with our previous finding on baicalein anti-dengue activity with $\mathrm{IC}_{50}=6.46 \mu \mathrm{g} / \mathrm{ml}^{9}$. Although, here we showed that baicalin exhibited intracellular antiviral activity against DENV-2 with $\mathrm{IC}_{50}=13.50 \mu \mathrm{g} / \mathrm{ml}$ its SI value however, remained 21.5, which is more significant as compared to its counterpart value for baicalein $(\mathrm{SI}=17.8)^{9}$. 


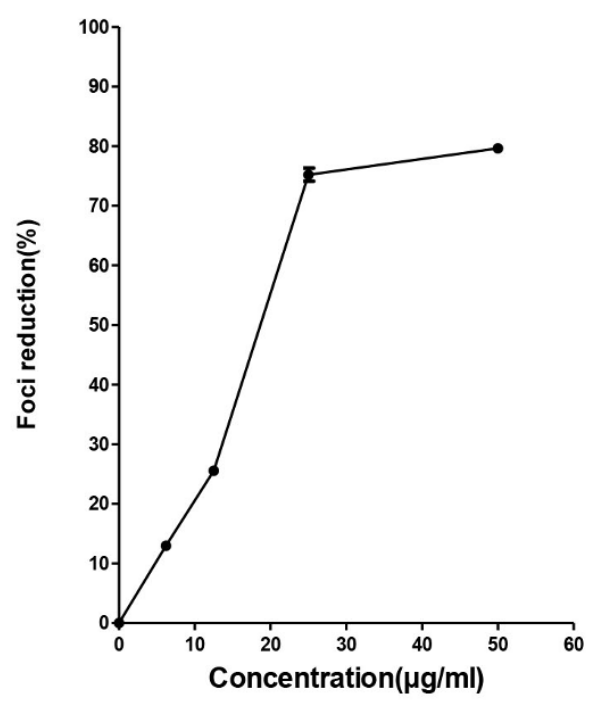

(A)

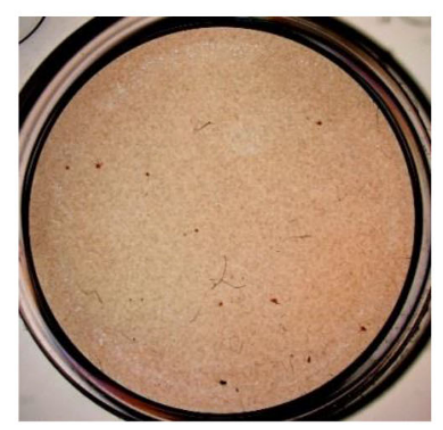

(B)

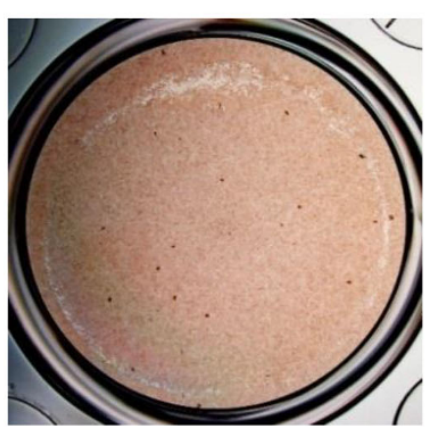

(C)

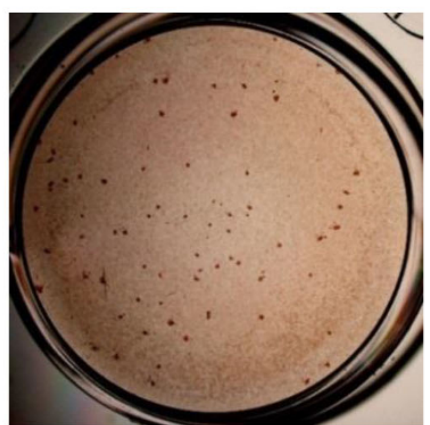

(D)

Figure 6 Effect of baicalin against DENV-2 cell attachment. Foci forming unit reduction assay (FFURA) was performed to evaluate the anti-attachment activity of baicalin against DENV attachment to the Vero cells. Anti-attachment activity of $50 \mu \mathrm{g} / \mathrm{ml}$ of baicalin (B); or with $25 \mu \mathrm{g} / \mathrm{ml}$ of baicalin (C) or without baicalin (D) using DENV foci immunostaining method on the day 4 post infection were shown. Up to $50 \mu \mathrm{g} / \mathrm{ml}$ of baicalin used in this test and it was shown that the highest concentration of the baicalin can inhibit the DENV-2 cell attachment significantly (A). Data from triplicate assays for three independent experiments were plotted using Graph Pad Prism Version 5 (Graph Pad Software Inc., San Diego, CA.).

On the other hand, results of the virus inactivation assay suggests that baicalin could interact with extracellular DENV particles and inactivate them with SI value equal to 33.2. Accordingly, we recently showed that the extract of S.baicalensis and pure baicalein also exerted direct virucidal activity against DENV extracellular particles at SI values equal to 9.5 and 74.3 , respectively ${ }^{9,24}$. This property might confer baicalein and baicalin the potentials to reduce circulating DENV particles during viremic phase of the disease, which is important to minimize disease severity. Besides, it also significantly diminishes viral transmission from infected individuals to mosquitoes during blood meal.

Our data also suggest that DENV infection was significantly impaired if baicalin is present at the time of adsorption (SI $=16$ ). However, baicalin did not affect viral internalization following adsorption to the cell surface. These results suggest that baicalin may exert its antiviral activity by inactivation of virus particles at high concentrations and possibly by interference with DENV adsorption to Vero cells at non-virucidal concentrations. However, the precise mechanism by which baicalin inhibits the DENV binding process remains to be elucidated.

Owing to the influence made by baicalin affecting the different stages of DENV replication, further studies may be required to reveal the specific antiviral target(s) of the compound. Therefore, we currently continue our efforts to investigate the molecular and intracellular pathways the compound targets, especially especially identification of target viral genes and cellular elements such as cytokines that could play a role in facilitating its anti-DENV functions.

In conclusion, we convincingly showed that baicalin the main metabolite of baicalein, a dengue virus replication inhibitor also exerts a antiviral activity against DENV-2 in vitro replication. Baicalin interferes with intracellular virus replication besides inactivation of free DENV particles and affects viral attachment step of DENV to host cells. Insights into the precise mechanisms whereby baicalin exerts its anti-dengue activity could lead to rational designing of more effective and selective inhibitors of DENV. Our finding of antiviral properties of baicalin supports further investigations directed on anti-DENV drug development.

\section{Methods}

Cells and virus. C6/36 mosquito cells and Vero (African green monkey kidney) cells (ATCC) were cultured and maintained in Eagle's Minimum Essential Medium (EMEM, Gibco, NY, USA) supplemented with 10\% fetal bovine serum (FBS) (Gibco, NY, USA). C6/36 and Vero cells were incubated at $28^{\circ} \mathrm{C}$ and $37^{\circ} \mathrm{C}$, respectively in a $5 \% \mathrm{CO} 2$ humidified atmosphere. BHK-DENV replicon cell line ${ }^{28}$ was cultured and maintained in Dulbecco's Minimum Essential Medium (DMEM, Gibco, NY, USA) containing $2 \%$ FBS and $1 \mathrm{mg} / \mathrm{ml} \mathrm{G} 418$. 


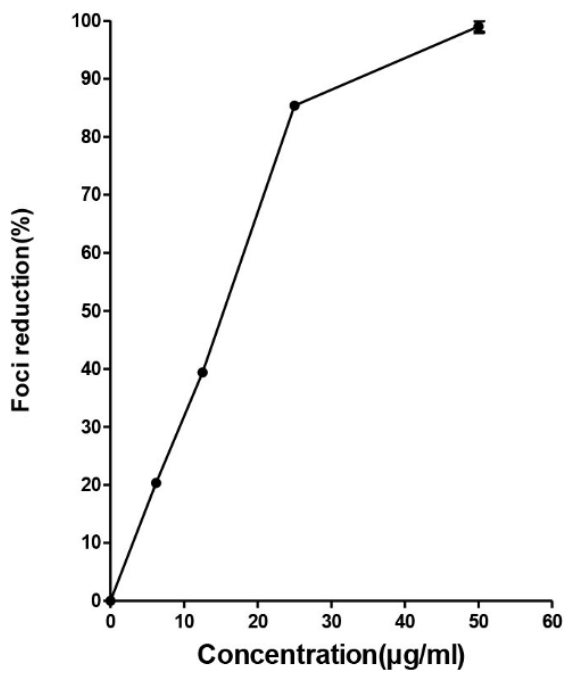

(A)

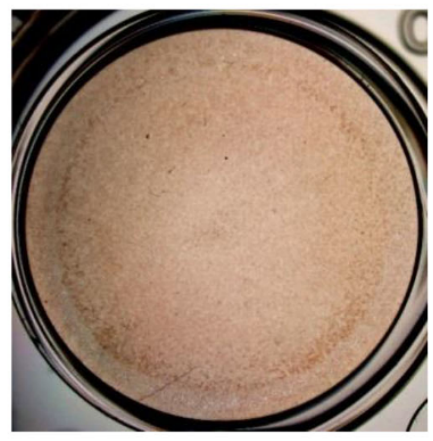

(A)

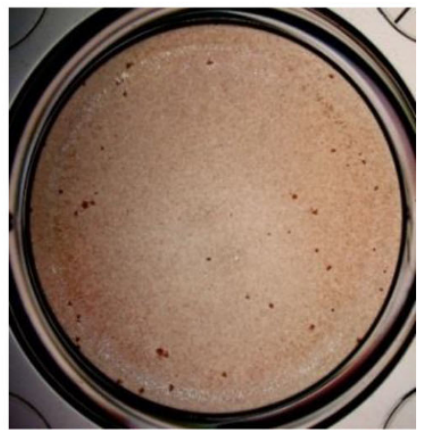

(B)

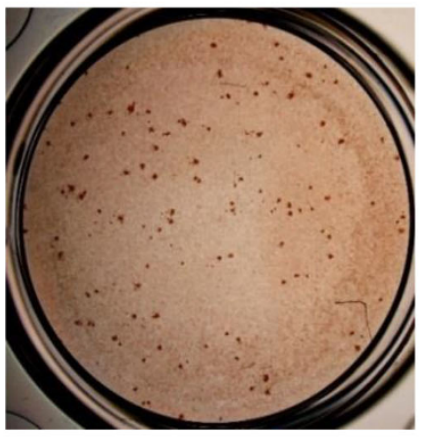

(C)

Figure $7 \mid$ Antiviral activity of baicalin against DENV after virus internalization. Foci forming unit reduction assay (FFURA) on Vero cells was performed to determine the antiviral activity of baicalin on DENV-2 intracellular replication after virus entry to the cells. Intracellular antiviral activity of $50 \mu \mathrm{g} / \mathrm{ml}$ of baicalin (B); or with $25 \mu \mathrm{g} / \mathrm{ml}$ of baicalin (C) or without baicalin (D) using DENV foci immunostaining method on the day three post infection were shown. It was shown that $50 \mu \mathrm{g} / \mathrm{ml}$ of baicalin inhibited the DENV-2 in vitro replication with $99 \%$ efficacy compared to the non-treated infected cells (A). Data from triplicate assays for three independent experiments were plotted using Graph Pad Prism Version 5 (Graph Pad Software Inc., San Diego, CA.).

In this study dengue virus type-2 (DENV-2) New Guinea C strain (NGC) was used. The virus was maintained at the Virology laboratory of the Tropical Infectious Disease Research and Education Center (TIDREC), Faculty of Medicine, University of Malaya (Kuala Lumpur, Malaysia). C6/36 cells were infected with DENV-2 and supernatant consisting of viruses were harvested after observation of cytopathic effect (CPE) on day seven post infection. Virus stock to the study was prepared and titrated on Vero cells using the focus forming assay as previously described ${ }^{29}$ and stored at $-80^{\circ} \mathrm{C}$ until needed. At the time of virus propagation and antiviral assays, the concentration of FBS was reduced to $2 \%$.

Flavonoid. Baicalin was purchased from Sigma Chemical Company (Sigma, St Louis, USA). The stock solution $(50 \mathrm{mg} / \mathrm{ml}$ ) was prepared in Dimethyl sulfoxide (DMSO) (Sigma-Aldrich, St. Louis, MO, USA) and stored at $-20^{\circ} \mathrm{C}$ until needed. At the time of experiment, the stock solution was serially diluted using EMEM (Gibco, NY, USA), containing $2 \%$ fetal bovine serum (FBS) and sterilized with syringe filter $0.2 \mu \mathrm{m}$ pore size (Millipore, MA, USA).

Cell cytotoxicity assay. Cytotoxicity of baicalin against Vero cells was determined using the MTT assay as previously describe $e^{30}$. Briefly, a confluent monolayer of Vero cells in 96-well cell culture microplate were treated with increasing concentrations of baicalin in triplicates for 4 days equivalent to that used in the antiviral assays. After 4 days, MTT solution (Promega, Madison, WI, USA) $(15 \mu \mathrm{l})$ was added to each well and the microplate was kept at $37^{\circ} \mathrm{C}$ for $4 \mathrm{~h}$ in a humidified atmosphere with $5 \% \mathrm{CO}_{2}$. Then, solubilization/stop solution $(100 \mu \mathrm{l})$ was added to the wells and the absorbance values of the wells were measured at $570 \mathrm{~nm}$ using a 96-well plate reader (TECAN, Mannendorf, Switzerland). Dose-response curve was plotted using Graph Pad Prism
5 (Graph Pad Software Inc., San Diego, CA, USA, 2005) and the half maximal cytotoxic concentration $\left(\mathrm{CC}_{50}\right)$ of baicalin was determined from the plot. Results were represent as the means \pm standard error of the mean (SEM) from triplicate assay from three independent experiments.

DENV replicon cell system. BHK-DENV replicon cells ${ }^{28}$ were plated at a density of $1 \times 10^{4}$ cells/well in a tissue culture-treated white view 96-well plate (Promega). The following day, the all culture medium was replaced with DMEM containing of $2 \%$ FBS and increasing dilution of baicalin. Treated cells were then incubated for $48 \mathrm{~h}$ at $37^{\circ} \mathrm{C}$ in a $5 \% \mathrm{CO}_{2}$ humidified chamber. The culture medium was decaned and cells were rinsed with PBS. Cells were lysed with $100 \mu \mathrm{l}$ of lysis buffer (Promega), and the luciferase activity was evaluated following the manufacturer's protocol (Promega). Triplicate wells were lysed at the time indicated and luminescence signal measured using the GloMAX 20/20 Luminometer (Promega). The luminescence signal was plotted against the log transformation of the concentration of baicalin and a sigmoidal curve fit with variable slope was created to obtain the half maximal inhibitory concentration $\left(\mathrm{IC}_{50}\right.$ ) value using Graph Pad Prism 5 (Graph Pad Software Inc., San Diego, CA, USA, 2005). Results were represent as the means \pm standard error of the mean (SEM) from triplicate assay from three independent experiments.

Quantitative reverse transcriptase polymerase chain reaction (qRT-PCR). Extracellular DENV-2 RNAs were extracted from the supernatant of the DENV-2 infected cells using the RNA extraction kit (Qiagen, Hilden, Germany). The quantitative RT-PCR was performed by adding $1 \mu \mathrm{l}$ of extracted DENV-2 RNA to the SensiMix SYBR green mixture (Quantace, Watford, United Kingdom) together with 
$50 \mathrm{pmol}$ of forward (DNF) and also reverse (D2R) primers ${ }^{31}$. Amplification was performed using the DNA Engine Opticon system (MJ Research/Bio-Rad, Hercules, CA) with the following thermal cycling conditions; reverse transcription at $50^{\circ} \mathrm{C}$ for $30 \mathrm{~min}$, initial denaturation at $95^{\circ} \mathrm{C}$ for $10 \mathrm{~min}$, followed by 45 cycles of $95^{\circ} \mathrm{C}$ for $15 \mathrm{sec}, 59^{\circ} \mathrm{C}$ for $30 \mathrm{sec}$ and $72^{\circ} \mathrm{C}$ for $30 \mathrm{sec}$. Melting curve analysis was subsequently performed at temperature from $60^{\circ} \mathrm{C}$ to $98^{\circ} \mathrm{C}$ to verify the assay specificity. The absolute quantities of viral RNA in the samples were measured with a standard curve generated with a 5 -fold serially diluted viral RNA extracted from DENV-2 virus inoculum of known infectious titer.

Focus forming unit reduction assay (FFURA). Antiviral activity of baicalin was determined and evaluated by measuring the reduction in the number of DENV infectious foci after treatment. Briefly, infected Vero cells which were treated with different regimes were incubated for 4 days post infection (PI) using conditionedgrowth medium supplemented with $2 \%$ FBS and $1.5 \%$ carboxymethyl cellulose (CMC).Virus foci were visualized as previously described ${ }^{9}$. The number of DENV-2 foci was counted using a stereomicroscope and the virus titer was expressed as Foci Forming-Unit (FFU). Antiviral activities of the compounds were determined by calculating the percentage of foci reduction (\%RF) compared against the controls maintained in parallel using the following formula; $R F(\%)=(C-T) \times 100 / C$, where, $\mathrm{C}$ is the mean of the number of foci from triplicates treatment without compound added (vehicle control) and $\mathrm{T}$ is the mean of the number of foci from triplicates of each treatment measures with the respective compound ${ }^{32}$. Results were represent as the means \pm standard error of the mean (SEM) from triplicate assay from three independent experiments.

Virus yield reduction assay. Vero cells were seeded in 24-well cell culture microplates. A day later, confluent monolayers were overlaid with $100 \mu$ l of EMEM containing $2 \%$ FBS together with increasing concentrations of baicalin and $100 \mu$ lof DENV-2 (NGC) suspension $(\mathrm{moi}=0.1)$. After virus adsorption, cells were washed 3 times with PBS to remove unabsorbed viruses and then the cells were further treated for 3 days with baicalin. Then the supernatant was harvested daily and the quantity of DENV RNA was determined using the real-time quantitative RT-PCR up to day 3 post infection. However, the antiviral effect of baicalin was investigated using FFURA on the day 3 post infection as well. Acyclovir (Sigma-aldrich Co, St.Louis, MO) at $1 \mu \mathrm{M}$ was used as a non-flavivirus inhibitor throughout the period of treatment for vehicle control.

Time of addition studies. Prophylactic treatment. In order to determine the prophylactic effects of baicalin against dengue virus replication, different concentrations of baicalin were added to the confluent Vero cells in 24-wells microplate 5 hours prior to virus infection. The treatment medium was removed and the treated cells were washed twice with PBS. The cells were then infected with $200 \mathrm{FFU}$ of DENV-2 and incubated at $37^{\circ} \mathrm{C}$ for 4 days in the presence of $5 \% \mathrm{CO}_{2}$. After 4 days of infection, antiviral activity was determined by the reduction in foci count as previously described.

Anti-adsorption activity. The activity of baicalin against adsorption of DENV-2 to the Vero cells was measured by inoculating the confluent Vero monolayers in 24-wells cell culture microplate with $200 \mathrm{FFU}$ of DENV-2 in the presence or absence of different concentrations of baicalin and incubated at $4{ }^{\circ} \mathrm{C}$ for 1 hour for virus adsorption. Then the cells were washed with sterile PBS twice and overlaid with 1.5\% CMC containing EMEM with 2\% FBS. After 4 days of incubation, anti-adsorption activity of baicalin was determined by determining the reduction in foci numbers as described earlier.

Virus internalization inhibition. The effect of baicalin against DENV-2 internalization was investigated by adding $200 \mathrm{ffu}$ of DENV-2 in triplicate to $4{ }^{\circ} \mathrm{C}$ prechilled Vero cell monolayers. The microplate was incubated at $4^{\circ} \mathrm{C}$ for $1 \mathrm{~h}$ to virus attachment. After $1 \mathrm{~h}$ adsorption, unabsorbed viruses were removed and cells were washed with PBS and incubated at $37^{\circ} \mathrm{C}$ for $1 \mathrm{~h}$ in the presence or absence of different concentrations of baicalin. Then, cells were washed with PBS and treated with $0.1 \mathrm{ml}$ of citrate buffer (Citric acid $40 \mathrm{mM}, \mathrm{KCl} 10 \mathrm{mM}, \mathrm{NaCl} 135 \mathrm{mM}, \mathrm{pH}$ 3) for $1 \mathrm{~min}$ to inactivate adsorbed viruses but not internalized. The cells were overlaid with maintenance medium containing $1.5 \% \mathrm{CMC}$ and incubated at $37^{\circ} \mathrm{C}$ for 4 days. After 4 days of incubation, the effect of baicalin against virus internalization to the cells was determined by the reduction in foci numbers as described above.

Virus inactivation assay. A viral suspension containing $10^{5} \mathrm{FFU}$ of DENV-2 $(\mathrm{MOI}=$ 5) incubated with equal volume of the different concentrations of baicalin for $2 \mathrm{~h}$ at $37^{\circ} \mathrm{C}$. Then, Vero cells were infected with the 1000 fold diluted treated viral suspension in triplicates. After $1 \mathrm{~h}$ adsorption at $37^{\circ} \mathrm{C}$, cells were washed twice with PBS. Cells were overlaid by $1.5 \%$ CMC containing EMEM with $2 \%$ FBS and incubated at $37^{\circ} \mathrm{C}$ for 4 days. After 4 days of incubation, direct virucidal activity of baicalin was determined by reduction in foci numbers as described earlier.

Statistical analysis. Graph Pad Prism for Windows, Version 5 (Graph Pad Software Inc., San Diego, CA, 2005) was used to determine the half maximal cytotoxic concentration $\left(\mathrm{CC}_{50}\right)$ and half maximal inhibitory concentration $\left(\mathrm{IC}_{50}\right)$ values of baicalin. All $\mathrm{IC}_{50}$ and $\mathrm{CC}_{50}$ values were caculated as the means \pm standard error of the mean (SEM) from triplicate assay from three independent experiments. Selectivity Index value (SI) was determined as the ratio of $\mathrm{CC}_{50}$ to $\mathrm{IC}_{50}$.

1. Hidari, K. I. et al. Structure and anti-dengue virus activity of sulfated polysaccharide from a marine alga. Biochem. Biophys. Res. Commun 376, 91-95 (2008).

2. Talarico, L. B. \& Damonte, E. B. Interference in dengue virus adsorption and uncoating by carrageenans. Virology 363, 473-485 (2007).

3. Zandi, K. et al. Flavone enhances dengue virus type-2 (NGC strain) infectivity and replication in Vero cells. Molecules 17, 2437-2445 (2012a).

4. Huh, S. U. \& Paek, K. H. Plant RNA binding proteins for control of RNA virus infection. Front Physiol 4, 397 (2013).

5. Kumar, S. \& Pandey, A. K. Chemistry and Biological Activities of Flavonoids: An Overview. ScientificWorldJournal 29, 2013:1650 (2013).

6. Lyu, S. Y., Rhim, J. Y. \& Park, W. B. Antiherpetic activities of flavonoids against herpes simplex virus type 1 (HSV-1) and type 2 (HSV-2) in vitro. Arch. Pharm. Res 28, 1293-301 (2005).

7. Evers, D. L. et al. Human cytomegalovirus-inhibitory flavonoids: studies on antiviral activity and mechanism of action. Antiviral Res. 68, 124-134 (2005).

8. Beecher, G. R. Overview of dietary flavonoids: nomenclature, occurrence and intake. J. Nutr 133, 3248-3254 (2003).

9. Zandi, K. et al. Novel antiviral activity of baicalein against dengue virus. $B M C$ Complement Altern Med 12, 1-9 (2012b).

10. Zandi, K. et al. Antiviral activity of four types of bioflavonoid against dengue virus type-2. Virol. J 8, 560 (2011).

11. Ozcelik, B., Orhan, I. \& Toker, G. Antiviral and antimicrobial assessment of some selected flavonoids. Z. Naturforsch. C. Biosci 61, 632 (2006).

12. Tian, S. et al. Pharmacokinetic study of baicalein after oral administration in monkeys. Fitoterapia 83, 532-540 (2012).

13. Tian, S. et al. Pharmacokinetic study of baicalein and its major metabolites after iv administration in dogs. CHM 3, 196-201 (2011).

14. Che, Q. et al. Studies on metabolites of baicalin in human urine. Zhongguo Zhong Yao Za Zahi 26, 768 (2001)

15. Xu, G., Dou, J., Zhang, L., Guo, Q. \& Zhou, C. Inhibitory effects of baicalein on the influenza virus in vivo is determined by baicalin in the serum. Biol. Pharm. Bul 33 , 238-243 (2010).

16. Lai, M. Y., Hsiu, S. L., Chen, C. C., Hou, Y. C. \& Chao, P. D. L. Urinary pharmacokinetics of baicalein, wogonin and their glycosides after oral administration of Scutellariae Radix in humans. Biol. Pharm. Bull 26, 79-83 (2013).

17. Dou, J. et al. Effects of baicalein on Sendai virus in vivo are linked to serum baicalin and its inhibition of hemagglutinin-neuraminidase. Arch. Virol 156, 793-801 (2011).

18. Bhatt, S. et al. The global distribution and burden of dengue. Nature 496, 504-507 (2013).

19. Lim, S. P. et al. Ten years of dengue drug discovery: progress and prospects. Antiviral Res. 100, 500-19 (2013).

20. Koishi, A. C., Zanello, P. R., Bianco, É. M., Bordignon, J. \& Nunes Duarte dos Santos, C. Screening of dengue virus antiviral activity of marine seaweeds by an In Situ Enzyme-Linked Immunosorbent Assay. PLoS one 7, e51089 (2012).

21. Michel, T., Halabalaki, M. \& Skaltsounis, A. L. New concepts, experimental approaches, and dereplication strategies for the discovery of novel phytoestrogens from natural sources. Planta Med 79, 514-32 (2013).

22. Romano, B. et al. Novel insights into the pharmacology of flavonoids. Phytother Res 27, 1588-96 (2013)

23. Cushnie, T. P. \& Lamb, A. J. Antimicrobial activity of flavonoids. Int. J. Antimicrob. Agents 26, 343-56 (2005).

24. Zandi, K. et al. Extract of Scutellaria baicalensis inhibits dengue virus replication. BMC Complement Altern Med 13, 91(2013).

25. Guo, X. Y. et al. Identification of the metabolites of baicalein in human plasma. J Asian Nat Prod Res 13, 861-868 (2011).

26. Chen, S. et al. Effects of the flavonoid baicalin and its metabolite baicalein on androgen receptor expression, cell cycle progression and apoptosis of prostate cancer cell lines. Cell Prolif 34, 293-304 (2001).

27. Xin, W. et al. Research progress on pharmacological actions and mechanism of baicalein and baicalin. Curr Opin Complement Alternat Med 1, e00010 (2014).

28. Yang, C. C. et al. Characterization of an efficient dengue virus replicon for development of assays of discovery of small molecules against dengue virus. Antiviral Res. 98, 228-41(2013).

29. Wong, S. S., Abd-Jamil, J. \& Abubakar, S. Antibody neutralization and viral virulence in recurring dengue virus type 2 outbreaks. Viral Immunol 20, 359-368 (2007).

30. Wong, P. F. \& Abubakar, S. High intracellular $\mathrm{Zn}^{2+}$ ions modulate the VHR, ZAP 70 and ERK activities of LNCaP prostate cancer cells. Cell. Mol. Biol. Lett 13, 375-390 (2008)

31. Seah, C. L. K., Chow, V. T. K., Tan, H. C. \& Can, Y. C. Rapid, single-step RT-PCR typing of dengue viruses using five NS3 gene primers. J. Virol. Methods 51, 193-200 (1995). 
32. Laille, M., Gerald, F. \& Debitus, C. In vitro antiviral activity on dengue virus of marine natural products. Cell. Mol. Life. Sci. 54, 167-70 (1998).

\section{Acknowledgments}

The authors would like to thank the Ministry of Higher Education (MOHE), Malaysia, for High Impact Research (HIR) MOHE Grant (E000087-20001) and Long-Range Grant

Scheme (LRGS) LR001/2011F. They also would like to thank the University of Malaya for University Malaya Research Grant (UMRG) (RG383-11HTM).

\section{Author contributions}

Conceived and designed the experiments: K.Z., S.A.B. Performed the experiments: E.M., B.T.T., P.H., S.S.S., R.L. Analyzed the data: K.Z., S.S.B., A.Y., E.M., Z.C. Contributed essential reagents: A.Y. Wrote the manuscript: E.M., K.Z., A.Y., S.A.B. All authors reviewed the manuscript.

\section{Additional information}

Competing financial interests: The authors declare no competing financial interests.

How to cite this article: Moghaddam, E. et al. Baicalin, a metabolite of baicalein with antiviral activity against dengue virus. Sci. Rep. 4, 5452; DOI:10.1038/srep05452 (2014).

(c) (1) (2) This work is licensed under a Creative Commons Attribution-NonCommercialShareAlike 4.0 International License. The images or other third party material in this article are included in the article's Creative Commons license, unless indicated otherwise in the credit line; if the material is not included under the Creative Commons license, users will need to obtain permission from the license holder in order to reproduce the material. To view a copy of this license, visit http:// creativecommons.org/licenses/by-nc-sa/4.0/ 